\title{
Notes on scrambling in conformal field theory
}

\author{
Chang Liu* and David A. Lowe ${ }^{\dagger}$ \\ Physics Department, Brown University, Providence, Rhode Island 02912, USA
}

(Received 12 October 2018; published 26 December 2018)

\begin{abstract}
The onset of quantum chaos in quantum field theory may be studied using out-of-time-order correlators at finite temperature. Recent work argued that a time scale logarithmic in the central charge emerged in the context of two-dimensional conformal field theories, provided the intermediate channel was dominated by the Virasoro identity block. This suggests a wide class of conformal field theories that exhibit a version of fast scrambling. In the present work we study this idea in more detail. We begin by clarifying to what extent correlators of wave packets built out of superpositions of primary operators may be used to quantify quantum scrambling. Subject to certain caveats, these results concur with previous work. We then go on to study the contribution of intermediate states beyond the Virasoro identity block. We find that at late times, time-ordered correlators exhibit a familiar decoupling theorem, suppressing the contribution of higher dimension operators. However this is no longer true of the out-of-time-order correlators relevant for the discussion of quantum chaos. We compute the contributions of these conformal blocks to the relevant correlators and find they are able to dominate in many interesting limits. Interpreting these results in the context of holographic models of quantum gravity, sheds new light on the black hole information problem by exhibiting a class of correlators where bulk effective field theory does not predict its own demise.
\end{abstract}

DOI: $10.1103 /$ PhysRevD.98.126013

\section{INTRODUCTION}

It has been suggested that quantum theories of gravity exhibit a property known as fast scrambling, where a generic quantum state exhibits global thermalization in a time scale that is logarithmic in the system size [1]. It is interesting to explore this idea in the context of holographic theories of gravity dual to conformal field theories, where one may try to extract constraints on the class of conformal field theories with gravity duals.

One simple way to quantify this notion of scrambling is to consider the norm (or equivalently the square) of the commutator of a pair of Hermitian operators $V$ and $W$ at different times. For the purposes of the present paper, we will also consider the system at finite temperature, with an inverse temperature $\beta$. This leads to a relation with out-oftime-order correlators

\footnotetext{
*chang_liu3@brown.edu

lowe@brown.edu
}

Published by the American Physical Society under the terms of the Creative Commons Attribution 4.0 International license. Further distribution of this work must maintain attribution to the author(s) and the published article's title, journal citation, and DOI. Funded by SCOAP ${ }^{3}$.

$$
\begin{aligned}
-\left\langle[V(0), W(t)]^{2}\right\rangle_{\beta}= & \langle V(0) W(t) W(t) V(0)\rangle_{\beta} \\
& +\langle W(t) V(0) V(0) W(t)\rangle_{\beta} \\
& -\langle W(t) V(0) W(t) V(0)\rangle_{\beta} \\
& -\langle V(0) W(t) V(0) W(t)\rangle_{\beta} .
\end{aligned}
$$

For sufficiently late times, the first two terms are simply the time-independent disconnected diagram $\langle W W\rangle_{\beta}\langle V V\rangle_{\beta}$, while the last two terms are genuine out-of-time-order correlators. For the 2D conformal field theories of interest here, these correlators may be computed by continuing the Euclidean four-point function through the second Riemann sheet [2], as we describe in detail later. These terms vary as a function of $t$, unlike the disconnected terms, and from them a scrambling time scale may be extracted. In the following section we describe in more detail the dependence of this time scale on the chosen operators. Briefly, one wishes to choose operators that exhibit the longest scrambling time scale, so one may use this commutator computation as a proxy for asking that the longest time scale a generic state scrambles. There may of course exist special choices of operators with much shorter scrambling times, and likewise special choices with much longer times, such as those that commute with the Hamiltonian.

In order to study these out-of-time-order correlators at finite temperature in conformal field theory (CFT) we will 
begin with the Euclidean theory on $S^{1} \times \mathbb{R}$. The correlators in this theory may be obtained by a conformal mapping from the complex plane. The circle direction is to be periodically identified with period $\beta$ and corresponds to the imaginary time direction. The spatial direction is then necessarily of an infinite extent. For the purposes of the present paper we will study four-point correlators of primary operators, as well as correlators of wave packets of such operators. Four-point functions of primaries are expressed in the so-called conformal blocks of the theory. In general, these conformal blocks are not known beyond infinite series expansions. However there has been much progress in the literature on obtaining asymptotic expansions of these conformal blocks in a variety of limits, and we will make extensive use of these results in the following [3].

In holographic theories, the graviton mode is dual to the stress energy tensor of the CFT, which in turn is a Virasoro descendant of the identity operator. Long distance bulk physics should be dominated by the propagation of this mode, so the limit where the identity block dominates the conformal block is of particular interest. Assuming the identity Verma module dominates the conformal block of the four-point function (as well as assuming a large central charge and large external conformal weight $h_{w}$ ) [2] obtained a scrambling time logarithmic in the central charge $c$ of the CFT,

$$
t_{*}=\frac{\beta}{2 \pi} \log \frac{c}{h_{w}},
$$

suggesting (at least if the result can be continued to values $h_{w}$ of order 1) that conformal field theories exhibit a version of fast scrambling. Closely related calculations were also performed in [4-6] using the vanishing of the mutual information to define the scrambling time. Potentially, this is a more sensitive diagnostic, since it avoids having to try to choose a "generic" primary operator wave packet, as we discuss below (see also [7]). Modulo this subtlety, the results in the end agree, assuming domination of the identity block. In a similar vein, [8] computed the entanglement entropy in holographic CFTs (i.e., those with $c \gg 1$ ) and obtained a similar time scale logarithmic in the central charge, although with the additional assumption of weak coupling.

In this paper we will study the issue of scrambling in more detail. One immediate issue is that primary operators on their own do not exhibit the time scale (2), but rather a thermalization time scale of order $\beta$ or less. However the class of states obtained by acting on the thermal state with a primary is not necessarily a good representative of a generic state, so this is not an immediate contradiction. To proceed we fold the primary operators into wave packets and consider optimizing the shape of the wave packet to obtain the longest thermalization time. When this is done, we find a time scale resembling (2) does indeed emerge. Next we examine the contribution of Verma modules with higher conformal weights to the four-point function. While we find the time-ordered four-point functions respect the familiar decoupling theorems and can be ignored with respect to the identity block, this is no longer true of the out-of-time-order correlators needed to compute (1). We compute the contributions of these higher intermediate states and find these can indeed dominate the commutator even when all the timeordered correlators have a sensible holographic description in terms of bulk low energy effective field theory. This implies that many of the bulk observables, defined over finite ranges of time, that one might use to probe the black hole information problem, are not accessible using low energy effective field theory. In this sense effective field theory does not predict its own demise.

\section{SCRAMBLING AND CFT CORRELATORS}

We consider a thermal system described by a conformal field theory living on a spatial real line $x$ with imaginary time $-i t$ periodically identified with a period $\beta$. We can map this spatially infinite thermal system to a CFT defined on the complex plane $z$ via the exponential map

$$
z=\exp \left(\frac{2 \pi}{\beta}(x+t)\right)
$$

We are interested in computing the 4-point functions that appear in (1) so to this end we consider four pairwise local operators, inserted at distinct spatial positions as in Fig. 1. We therefore have, after conformal mapping,

$$
\begin{aligned}
& z_{1}=e^{\frac{2 \pi}{\beta} x_{1}} \\
& z_{2}=e^{\frac{2 \pi}{\beta} x_{2}} \\
& z_{3}=e^{\frac{2 \pi}{\beta}\left(x_{3}+t\right)} \\
& z_{4}=e^{\frac{2 \pi}{\beta}\left(x_{4}+t\right)},
\end{aligned}
$$

where we are interested in the limit $x_{1} \rightarrow x_{2}, x_{3} \rightarrow x_{4}$ to reproduce the desired commutator.

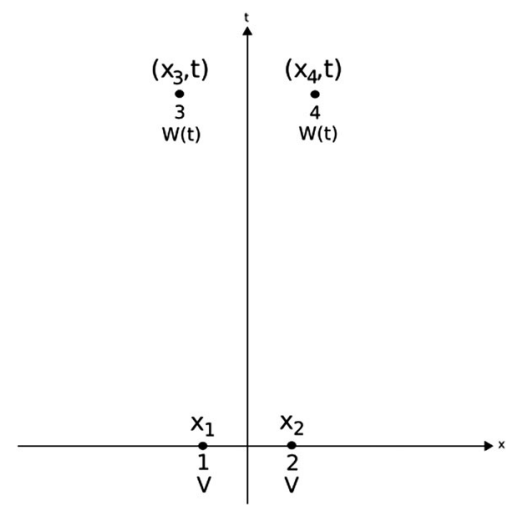

FIG. 1. The configuration of four pairwise identical local operators, themselves separated by large $t$. 


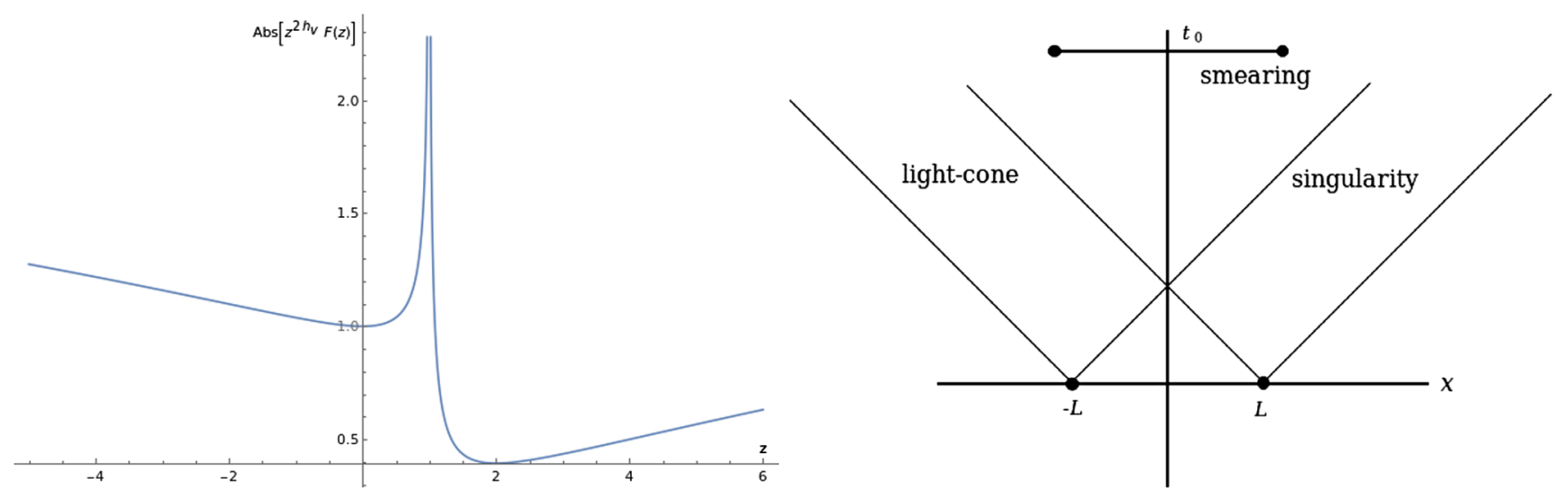

FIG. 2. (Left) Plot of the absolute value of the principal sheet rescaled identity block, which has a cusp at $z=1$. Due to the cusp the convolution of the rescaled identity block is dominated by its value near $z=1$. (Right) We work in the regime of $t>\beta$ and smear the operators within the light cone in order to avoid the light cone singularity of the conformal cross-ratio $z$. We fix the value of $L$ by maximizing the smeared cross-ratio at an initial time $t_{0}$ to find $L \approx \beta$ and use this (fixed) $L$ to compute the saddle point of $z$ at late-time.

The spacetime dependence of the conformal blocks appearing in the 4-point function will only depend on the cross-ratio $z=z_{12} z_{34} / z_{13} z_{24}$ (and $\bar{z}$ ) which is easily shown to be

$$
z=\frac{\sinh \left(\frac{\pi}{\beta}\left(x_{1}-x_{2}\right)\right) \sinh \left(\frac{\pi}{\beta}\left(x_{3}-x_{4}\right)\right)}{\sinh \left(\frac{\pi}{\beta}\left(t-x_{1}+x_{3}\right)\right) \sinh \left(\frac{\pi}{\beta}\left(t-x_{2}+x_{4}\right)\right)} .
$$

As discussed in the Appendix we rescale the 4-point function by the coincident 2-point functions, to scale out the operator norm. The rescaled correlators then depend only on the cross-ratios as in (A4).

As an example, let us consider the identity conformal block in a large $c$ limit, where the $V$ and $W$ operators have conformal weights $h_{v}$ and $h_{w}$ respectively. The large $c$ limit is to be taken with $h_{w} / c$ fixed, and $h_{v} \ll c$ fixed. The conformal block $\mathcal{F}(z)$ in this limit is computed in [3,9]

$$
z^{2 h_{v}} \mathcal{F}(z) \approx\left[\frac{z \alpha_{w}(1-z)^{\left(\alpha_{w}-1\right) / 2}}{1-(1-z)^{\alpha_{w}}}\right]^{2 h_{v}}
$$

with $\alpha_{w}=\sqrt{1-24 h_{w} / c}$. The real-time out-of-time-order correlator is obtained by continuing this block to the second Riemann sheet as described in [2] and the leading contribution to the rescaled commutator is

$$
\begin{aligned}
z^{2 h_{v}} \mathcal{F}(z) & \approx\left[\frac{e^{-\pi i\left(\alpha_{w}-1\right)} z \alpha_{w}(1-z)^{\left(\alpha_{w}-1\right) / 2}}{1-e^{-2 \pi i \alpha_{w}}(1-z)^{\alpha_{w}}}\right]^{2 h_{v}} \\
& \sim\left(\frac{1}{1-\frac{24 \pi i h_{w}}{c z}}\right)^{2 h_{v}} .
\end{aligned}
$$

Let us take a limit where $\epsilon_{12}=x_{1}-x_{2}$ and $\epsilon_{34}=x_{3}-x_{4}$ are much smaller than $\beta$, and without a loss of generality set $x_{1}=0$. The cross-ratio is then approximately

$$
z \approx \frac{\pi^{2}}{\beta^{2}} \frac{\epsilon_{12} \epsilon_{34}}{\sinh ^{2}\left(\frac{\pi}{\beta}\left(t+x_{3}\right)\right)}
$$

provided we stay away from lightlike separations where $x_{3} \rightarrow-t$. As we see the conformal block on the second sheet has a simple limit as $\epsilon_{12}$ and $\epsilon_{34} \rightarrow 0$, when $z \rightarrow 0$, corresponding to the actual computation of the commutator

$$
z^{2 h_{v}} \mathcal{F}(z) \approx\left(\frac{c z}{24 \pi i h_{w}}\right)^{2 h_{v}}
$$

The exponential decay of this quantity indicates the commutator between $V$ and $W$ becomes large after a time of order

$$
t=\frac{\beta}{4 \pi h_{v}}
$$

showing rapid thermalization of primary operators on a time scale much shorter than (2).

However the interesting physical question is whether generic states exhibit some notion of quantum scrambling on a longer time scale. To explore this question in the current context of CFT 4-point functions, we can then try to build more generic deformations of the thermal density matrix by acting with primary operators folded into wave packets with some characteristic spatial size $L$. Computing the 4-point function of these wave packets, one can attempt to vary $L$ to maximize the convoluted amplitude, then ask what thermalization time scale emerges.

Concretely, we convolute the function (4) with spatial Gaussian wave packets with width $L$. For some choice of initial time $t_{0}$ as shown in Fig. 2 we choose to optimize $L$ to maximize the absolute value of the convolution. The convolution may then be well approximated by setting $z=1$. At later times, we keep the size $L$ of the wave 
packets fixed in the $(x, t)$ coordinates and note that the convolution at later times is again dominated the a particular value of the cross ratio, that we compute below.

For the physical problem at hand, we choose to avoid the light cone singularities indicated in Fig. 2, which are present in any amplitude derived using primary operators, and reflect the causal properties of the commutator, which bounds the range of interesting values of $L$. We also note the final answer for $z$ at the saddle will depend on $t_{0}$. However since the dependence on $t_{0}$ is rather trivial, we choose to simplify the presentation by extrapolating it to 0 .

For simplicity let us set $x_{1}+x_{2}=x_{3}+x_{4}=0$, and we will build Gaussian wave packets in the variables $x_{1}-x_{2}=$ $\ell_{v}$ and $x_{3}-x_{4}=\ell_{w}$. Using a leading order saddle point approximation to the convolution integral, it suffices to study the saddle point value of the cross ratio. Therefore, to fix $L$ in terms of $z$, we consider the integral

$$
\begin{aligned}
z(t, L)= & \frac{4}{\pi L^{2}} \int_{0}^{t-\epsilon} d l_{v} d l_{w} e^{-\left(l_{v}^{2}+l_{w}^{2}\right) / L^{2}} \\
& \times \frac{\sinh \left(\frac{\pi}{\beta} l_{v}\right) \sinh \left(\frac{\pi}{\beta} l_{w}\right)}{\sinh \left(\frac{\pi}{\beta}\left(t-\frac{l_{v}}{2}+\frac{l_{w}}{2}\right)\right) \sinh \left(\frac{\pi}{\beta}\left(t+\frac{l_{v}}{2}-\frac{l_{w}}{2}\right)\right)},
\end{aligned}
$$

where the limits of the integral indicate that we are staying within the light cone when doing the smearing. As we have explained in the previous paragraph, this formula is justified because the exponential variation of $z$ with $l_{v}, l_{w}$ is much more rapid than power law variation of the conformal block with $z$, so analyzing the convolution of $z$ alone is sufficient to determine $l_{v}$ and $l_{w}$ and subsequently $L$. The integrand has lightlike poles; however for suitable values of $t$ and $L$ these contributions to the smeared conformal block can be made negligible. In this limit, the integrand can be well-approximated by simply

$z(t, L) \approx \frac{4}{\pi L^{2}} \int_{0}^{t-\epsilon} d l_{v} d l_{w} e^{-\left(l_{v}^{2}+l_{w}^{2}\right) / L^{2}} \frac{2 \sinh \left(\frac{\pi}{\beta} l_{v}\right) \sinh \left(\frac{\pi}{\beta} l_{w}\right)}{\cosh \left(\frac{2 \pi}{\beta} t\right)}$,

taking $t \gg \beta$. This has saddle points when

$$
l_{v} \tanh \left(\frac{l_{v} \pi}{\beta}\right)=\frac{\pi L^{2}}{2 \beta},
$$

and likewise for $l_{w}$. The positive solutions are to be taken corresponding to the limits of integration in (7). If we then ask that the resulting amplitude (4) is maximized in magnitude, we find that we must choose $L \sim \beta$ near $t=t_{0} \gtrsim \beta$. Alternatively, one can see this by numerically evaluating the integral (8) in the regime we are interested in and see that $z(t, L)$ is maximized near $L \sim \beta$ for an initial time $t_{0} \gtrsim \beta$ (see Fig. 3). Keeping the wave packet size fixed in $(x, t)$ coordinates, and extrapolating $t_{0}$ to zero, leads to the saddle point value for general $t$

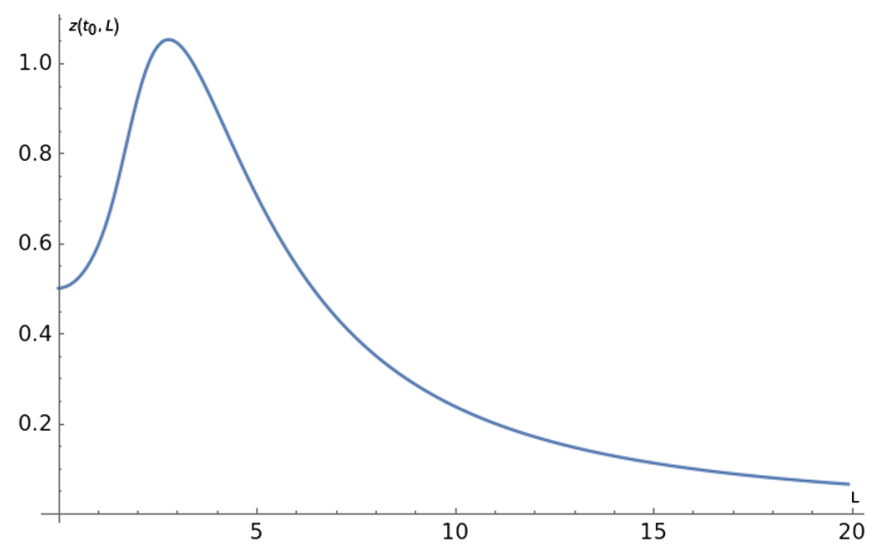

FIG. 3. Numerical integral of $z\left(t_{0}, L\right) \cosh \left(2 \pi t_{0} / \beta\right)$ in the regime $t_{0} \gtrsim \beta$, where we have set $\beta=\pi$. We see that the integral is maximized near $L \sim \beta$ and evaluates to the cusp value of $z=1$.

$$
z_{\mathrm{sad}} \propto \operatorname{sech}\left(\frac{2 \pi}{\beta} t\right) \approx e^{-2 \pi t / \beta},
$$

up to constant factors of order 1 .

Let us now return to the example of the identity conformal block continued to the second Riemann sheet as considered in [2]. In this case, the saddle point approximation to the (rescaled) convoluted block function is for sufficiently late times

$$
z^{2 h_{v}} \mathcal{F}(z) \approx\left(\frac{1}{1-\frac{12 \pi i h_{w}}{c} e^{\frac{2 \pi}{\beta}(t-x)}}\right)^{2 h_{v}},
$$

where we have restored dependence on the spatial separation $x$ of the centers of the wave packets, and inserted the saddle point approximation value for $z$ (9) for $t \gg \beta$. It is helpful to plot this for sample parameters as in Fig. 4. As $t-x$ increases from 0 to

$$
t_{*}=\frac{\beta}{2 \pi} \log \frac{c \sqrt{\log 2}}{12 \pi h_{v}^{1 / 2} h_{w}},
$$

the conformal block decreases in magnitude by a factor of about $1 / 2$. This thermalization time may be viewed as a proxy for the true scrambling time of the system and shows the distinctive appearance of the logarithm of the system size. Ideally one would want to argue this formula continues to hold as $h_{v}$ and $h_{w}$ become of order 1, but it is not yet possible to prove this. We note there are minor disagreements with the formula presented in [2]. We note Fig. 4 also shows in the late-time limit the asymptotic form (5) is applicable and the time scale for variation is the much shorter time (6).

The correlator of the wave packets is given by (10) provided one steers clear of the light cone singularities in (7) which render the approximation (9) invalid. This is a signature that even the wave packets of primaries are not 


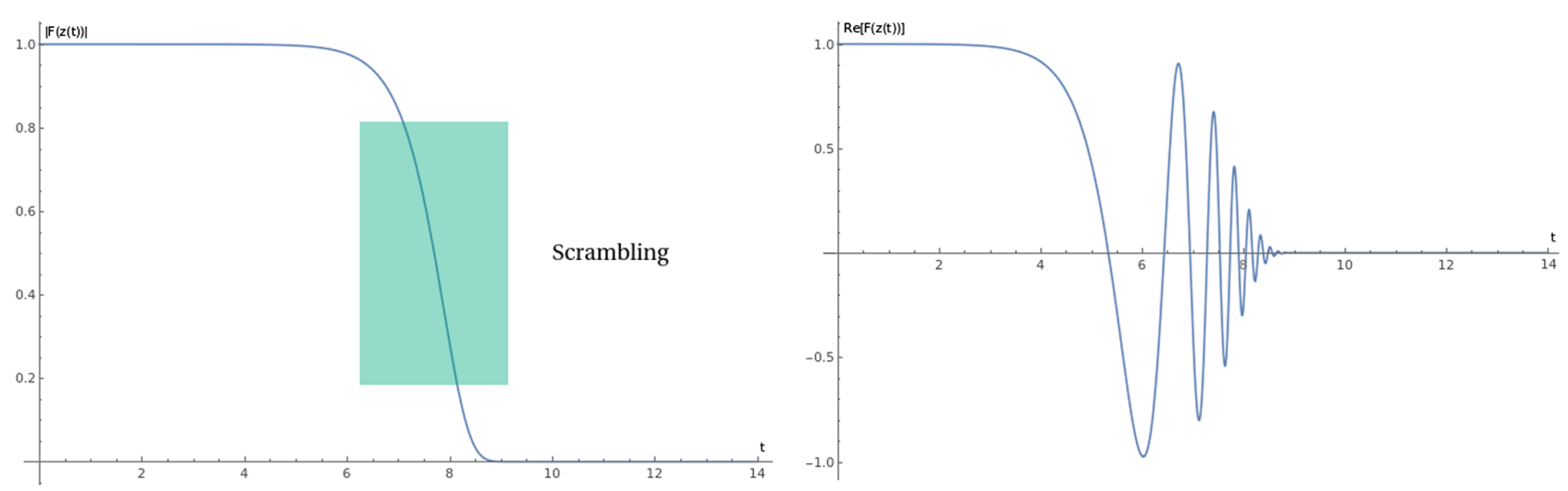

FIG. 4. Plot of function $\left|z^{2 h_{v}} \mathcal{F}(z)\right|=|F(z(t))|=1 /\left|1-12 \pi i h_{w} \exp (2 \pi / \beta(t-\log c-x))\right|^{2 h_{v}}$, where $c=10^{7}, h_{v}=100, h_{w}=10$, $\beta=2 \pi$ and $x=0$. Here $t_{*}=7.7$ according to (11). In the right panel, a plot of $\operatorname{Re} F(z(t))$ is shown.

ideal representatives of a generic state and retain regions of spacetime where thermalization has not yet occurred, outside the light cone of the wave packet. Nevertheless for the present purposes, the reduced state inside the light cone appears to be well-thermalized according to the correlators, so this procedure should yield a good measure of the global scrambling time. Again it remains to be seen whether (11) holds in the case of physical interest where $h_{v}$, $h_{w}$ are of order 1.

\section{HIGHER WEIGHT INTERMEDIATE STATES}

We now turn our attention to the contribution of higher weight intermediate states to the out-of-time order correlators and will find the surprising result that these may dominate over the identity block in the late-time limit. Again we will assume we are taking $c \gg 1$ with $h_{w} / c$ fixed and $h_{v} \ll c$ fixed. In addition we will generalize from the identity block to an intermediate channel with conformal weight $h_{p}$ fixed as $c \rightarrow \infty$.

Our starting point is the formula for the conformal block at next-to-leading order in this large $c$ expansion of [9]

$$
\begin{aligned}
& \mathcal{F}(z) \\
& =\mathcal{F}_{0}(z)\left(\frac{1-(1-z)^{\alpha_{w}}}{\alpha_{w}}\right){ }_{2}^{h_{p}} F_{1}\left(h_{p}, h_{p}, 2 h_{p}, 1-(1-z)^{\alpha_{w}}\right),
\end{aligned}
$$

where ${ }_{2} F_{1}(\alpha, \beta ; \gamma ; z)$ is the Gauss hypergeometric function. As we have mentioned in the Introduction section, the latetime behavior of the out-of-time-ordered correlator is given by analytically continuing the rescaled conformal block to the second Riemann sheet. More concretely, this means that we will circle $z$ around the branch cut $z=1$ of the rescaled conformal block $z^{2 h_{v}} \mathcal{F}(z)$ (see Fig. 5) where in this process $(1-z)^{\alpha_{w}}$ will gain an additional phase factor: $(1-z)^{\alpha_{w}} \rightarrow$ $e^{2 \pi i \alpha_{w}}(1-z)^{\alpha_{w}}$. As is pointed out in [2], this second sheet expression of the identity block gives the scrambling time scale $t_{\star} \sim \log c$.
To continue the conformal block (12) to the second sheet we use the hypergeometric function identity [10]

$$
\begin{aligned}
& \frac{\Gamma(h)^{2}}{\Gamma(2 h)^{2}}{ }_{2} F_{1}(h, h ; 2 h ; w) \\
& \quad=\left(\sum_{k=0}^{\infty} \frac{2(h)_{k}^{2}(\psi(k+1)-\psi(h+k))}{k !^{2}}(1-w)^{k}\right) \\
& \quad-\log (1-w)_{2} F_{1}(h, h, 1 ; 1-w)
\end{aligned}
$$

valid for $|1-w|<1$, where $(h)_{k}$ is the Pochhammer symbol, and $\psi(a)$ is the digamma function. We note that the infinite sum involving the digamma functions is analytic in $|1-w|<1$ and so is the hypergeometric function ${ }_{2} F_{1}(h, h, 1 ; 1-w)$ on the right-hand side of the above identity. Both of these have trivial behaviors on the second Riemann sheet. The only nonanalytic part is $\log (1-w)$, which when continued to the second sheet gives an additional term $2 \pi i \alpha_{w}: \log (1-w) \rightarrow \log (1-w)+$ $2 \pi i \alpha_{w}$ since as we have pointed out above $1-w=(1-z)^{\alpha_{w}}$ becomes $e^{2 \pi i \alpha_{w}}(1-z)^{\alpha_{w}}=e^{2 \pi i \alpha_{w}}(1-w)$ on the second sheet. The first term in $\log (1-w)+2 \pi i \alpha_{w}$ then combines

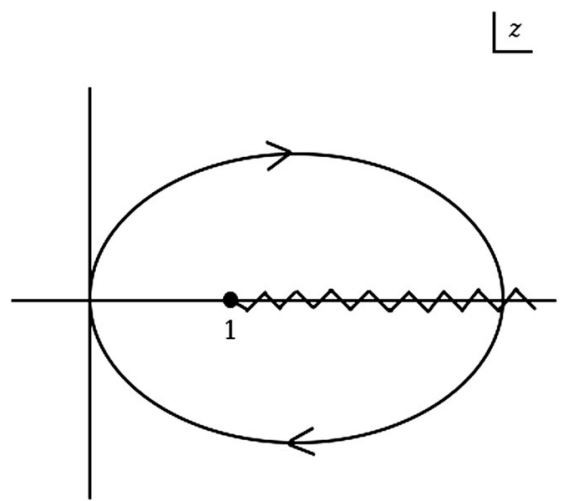

FIG. 5. Analytic continuation of the rescaled conformal block, where we circle around the branch cut $z=1$ to get to the second Riemann sheet. 
with the infinite sum to give the original principal sheet expression back. We conclude that continuing to the second Riemann sheet gives the original principal sheet hypergeometric function plus an additional term proportional to $2 \pi i \alpha_{w}$, namely

$$
\begin{aligned}
\mathcal{F}_{I I}(z)= & \mathcal{F}_{0, I I}(z)\left(\frac{1-e^{-i 2 \pi \alpha_{w}}(1-z)^{\alpha_{w}}}{\alpha_{w}}\right)^{h_{p}} \\
& \times\left({ }_{2} F_{1}\left(h_{p}, h_{p}, 2 h_{p}, 1-e^{-i 2 \pi \alpha_{w}}(1-z)^{\alpha_{w}}\right)\right. \\
& \left.+2 \pi i \alpha_{w} \frac{\Gamma\left(2 h_{p}\right)}{\Gamma\left(h_{p}\right)^{2}} 2 F_{1}\left(h_{p}, h_{p}, 1 ; e^{-i 2 \pi \alpha_{w}}(1-z)^{\alpha_{w}}\right)\right),
\end{aligned}
$$

where as usual the functions on the right-hand side of (13) are to be evaluated on the principal sheet. Expanding for small $h_{w} / c$ and $z \ll 1$ leads to

$$
\begin{aligned}
\mathcal{F}_{I I}(z) \sim & \mathcal{F}_{0, I I}(z)\left(\frac{z-\frac{\pi i h_{w}}{6 c}}{\alpha_{w}}\right)^{h_{p}} \\
& \times\left(1+i \tan \left(\pi h_{p}\right)-2 \pi^{2} i z^{1-2 h_{p}}\right. \\
& \left.\times \frac{\Gamma\left(2 h_{p}\right)}{\Gamma\left(2-2 h_{p}\right) \Gamma\left(h_{p}\right)^{4} \sin \left(2 \pi h_{p}\right)}\right) .
\end{aligned}
$$

This ends up being dominated by the last term in the third factor and in fact grows at late times. Even at early times ( $z$ near 1 ) the last term in (13) dominates over the other term in the third factor for $h_{p}>1$. The second factor in (13) rapidly approaches a constant much smaller than 1 .

The upshot is the identity block dominates for a finite period of time; however after

$$
t_{*} \approx \frac{\beta}{4 \pi} \log \left(\frac{c}{h_{w}}\right),
$$

the higher weight intermediate states take over. This late time sum over intermediate states apparently diverges when considered term by term. This would lead one to conclude the commutator grows initially while dominated by the identity block, but then may again decrease at later times, indicating a lack of true scrambling in the conformal field theory.

One possible way to avoid this conclusion is to demand an infinite tower of higher weight intermediate primaries, such that the apparently divergent sum might be resummed to a finite answer. To address this question would require additional specification of the conformal field theory being considered, so we do not consider it further in the present work.

For conformal field theories with holographic anti-de Sitter gravity duals, the implication of the higher intermediate channels is that the bulk effective field theory breaks down when it is used to compute out-of-timeordered correlators at finite time. On the other hand, there is no indication of such a breakdown when time-ordered CFT correlators are computed (see also $[11,12]$ ), which correspond to the boundary $S$-matrix of the bulk theory. To see this we simply note that as higher dimensional operators in $\mathrm{CFT}_{2}$ correspond to interactions of increasing mass scale in three-dimensional anti-de Sitter spacetime $\left(\mathrm{AdS}_{3}\right)$, domination of all intermediate channels with dimension $h_{p} \geq 1$ means that there would be a dual set of an infinite sequence of interactions in the gravitation theory in $\mathrm{AdS}_{3}$. If these high scale interactions affect the infrared physics of the theory, then the standard decoupling theorems of effective field theory such [13] break down.

Now the usual measurements we perform can be well-approximated by transition amplitudes, built out of time-ordered correlators which may be computed as within effective field theory. It is only the particular set of observables corresponding to out-of-time-order correlators or norms of commutators that exhibit this peculiar behavior. For the black hole information problem this would seem to imply that contrary to expectations, commutators that measure limits on the causal propagation of information are indeed observables sensitive to the ultraviolet structure of the theory, as long hinted at in perturbative string theory computations $[14,15]$.

Another consequence of this result is that thermalization of the operators we have considered may not actually happen. In light of our previous discussion, this may simply mean such smeared primaries are still not good representatives of generic states, and instead one would need to consider commutators of much more general operators to see the correct time scale for global thermalization or quantum scrambling.

Finally, [4,5] commented on the stringy effects in these out-of-time-ordered correlators and argued using perturbative bulk string theory computations that correct to the scrambling time become appreciable in a time of order $\beta / 2 \pi \log c$ in broad agreement with our result (14). However their main conclusion was the stringy corrections serve to only lengthen the scrambling time, preserving quantum chaos. In the context of the present work, such a conclusion would require additional assumptions, such as a large gap in the spectrum of primary operators, ensuring the intermediate states discussed here are not present.

\section{INTERMEDIATE CHANNELS WITH $h_{p} \gg c$}

So far we have only considered intermediate channels with fixed $h_{p} \ll c$. It is also instructive to perform the same analysis for intermediate channels with $h_{p} \gg c$ where the limit is $h_{p} \rightarrow \infty$ with $c / h_{p}, h_{v} / h_{p}$ and $h_{w} / h_{p}$ fixed and small. For this we consider Eq. (16) in [16], 


$$
\begin{aligned}
\mathcal{F}(z)= & (16 q)^{h_{p}-\frac{c}{24}} z^{\frac{c}{24}-2 h_{v}}(1-z)^{\frac{c}{24}-\left(h_{v}+h_{w}\right)} \\
& \times \theta_{3}(q)^{\frac{c}{2}-8\left(h_{v}+h_{w}\right)} H\left(c, h_{p}, h_{i}, q\right) .
\end{aligned}
$$

where the nome $q=e^{i \pi \tau}$ is related to the cross-ratio $z$ by

$$
\tau=i \frac{K^{\prime}(z)}{K(z)}=i \frac{K(1-z)}{K(z)},
$$

where $K(z)$ is the complete elliptic integral with parameter [17] $z$. Here $H$ is a function that is $1+O\left(1 / h_{p}\right)$ and

$$
\theta_{3}(q)=\sum_{n=-\infty}^{\infty} q^{n^{2}}
$$

Equation (15) has a branch cut at $z=1$ from the $1-z$ factor which will lead to the same analytic behavior for the intermediate case $h_{p} \ll c$, which we have previously considered. To see this we expand the nome $q$ around $z=0$ to obtain

$$
q=e^{i \pi \tau}=\frac{z}{16}+\frac{z^{2}}{32}+\cdots
$$

As $\theta_{3}(q)$ is regular near $q=0$, we see that on the principal sheet $\mathcal{F}(z)$ goes to zero as $z \rightarrow 0$. Therefore the heavy intermediate channels are perfectly suppressed on the first Riemann sheet. Crossing the branch cut $z=1$ from above, the complete elliptic function $K(z)$ picks up an additional imaginary part [18],

$$
\lim _{\epsilon \rightarrow 0^{+}} K(z+i \epsilon)=K(z)+2 i K(1-z) .
$$

Analyticity implies that on the second Riemann sheet the nome is now

$$
q=\exp \left[-\frac{\pi K(1-z)}{K(z)+2 i K(1-z)}\right]=\exp \left[-\frac{\pi}{\frac{K(z)}{K(1-z)}+2 i}\right] .
$$

To expand this expression near $z=0$, we use

$$
\frac{K(z)}{K(1-z)} \approx \frac{\pi}{4 \log 2-\log z}+\mathcal{O}\left(\frac{z}{\log ^{2} z}\right),
$$

so that

$$
q \approx e^{\frac{i \pi}{2}+\frac{\pi^{2}}{4 \log z}}
$$

We then need to expand (16) near $q=i$. The expansion near $q=1$ is

$$
\left|\theta_{3}(q)\right| \approx\left|\frac{\sqrt{\pi}}{\sqrt{1-q}}\right|
$$

but we can obtain the expansion near $q=i$ by using the relation

$$
\left|\theta_{3}(q)\right|=\left|\frac{\sqrt{\pi}}{\sqrt{\log q}} \theta_{3}\left(e^{\frac{\pi^{2}}{\log q}}\right)\right|
$$

and substituting in (17) to give $\theta_{3}(q)$ near $q=i$ as

$$
\left|\theta_{3}(q)\right| \approx\left|\frac{\sqrt{-2 \log z}}{\sqrt{\pi}}\right| .
$$

Assembling the various factors, we find again a dramatic enhancement of the higher weight channel on the second Riemann sheet arising from the behavior (18), compared to the behavior on the principal sheet. However when we compare to the $h_{p}=0$ expression of the previous section, the $z^{c / 24}$ factor of (15) dominates for small $z$ so we conclude they do not dominate vs the identity channel [again modulo restrictions on the operator couplings $C_{p}$ of (A2)].

\section{CONCLUSIONS}

In this paper we discussed the issue of smearing local operators in a thermal CFT and its connection with quantum scrambling. We pointed out that the correct scrambling time should be identified with operators that maximize the time scale of the variation of the out-of-time ordered correlator, which may occur well before the asymptotic late-time limit. We then examined a somewhat independent issue, that the higher intermediate states with $0<h_{p} \ll c$ can have large out-of-time ordered correlators. We discussed the implication of this statement, which is that in the $\mathrm{AdS}_{3}$ gravity dual the UV dynamics and IR dynamics is no longer decoupled when these observables are computed. This lack of decoupling appears even when the usual time-ordered correlators or transition amplitudes satisfy the standard decoupling lore. When applied to scattering in $\mathrm{AdS}_{3}$ black hole backgrounds this implies that the commutators that lead one to conclude information is lost semiclassically are in fact not computable without a full specification of the ultraviolet physics of the theory. The ordinary bulk effective field theory does not predict its own demise when computing these observables.

As for the appearance of a scrambling time of the form (2) we have found a variant of this expression (11) valid when the identity block dominates. The expression involves a term of the form $\beta / 2 \pi \log c$, but other significant terms are also present. If other intermediate primaries appear, with conformal weights fixed in a large $c$ limit, they will dominate the late-time behavior and may completely spoil thermalization. It will be very interesting to extend the range of validity of these expressions to determine whether there exist a class of $2 \mathrm{D}$ conformal field theories that may be viewed as fast scramblers at a finite temperature. 


\section{ACKNOWLEDGMENTS}

D. A. L. thanks S. Hellerman and E. Perlmutter for helpful comments. We thank B. Stoica, A. Rolph, and H. Hampapura for help in checking the semiclassical exponentiation formula for conformal blocks. We thank R. Fan for helpful comments on an earlier draft and note the closely related work in the context of $c<1$ unitary minimal models [19]. D. A. L. is supported in part by DOE Grant No. DESC0010010. This work was completed at the Aspen Center for Physics, which is supported by National Science Foundation Grant No. PHY-1607611.

\section{APPENDIX: CORRELATORS AND CONFORMAL BLOCKS}

Conformal blocks are usually written in terms of 4-point functions after a global $S L(2, C)$ conformal transformation has sent generic points in the complex plane to the values 0 , $z, 1, \infty$. Here we briefly unpack the relation between these conformal blocks and 4-point functions for general $z_{i}$.

A canonical form for the 4-point function at general $z_{i}$ in the complex plane is [20]

$$
\left\langle\prod_{i=1}^{4} \mathcal{O}_{i}\left(z_{i}\right)\right\rangle=f(z, \bar{z}) \prod_{i<j} z_{i j}^{-\left(h_{i}+h_{j}\right)+h / 3} \prod_{i<j} \bar{z}^{-\left(\bar{h}_{i}+\bar{h}_{j}\right)+\bar{h} / 3},
$$

where $z_{i j}=z_{i}-z_{j}$, the cross-ratio $z=z_{12} z_{34} / z_{13} z_{24}$ and $h=\sum_{i} h_{i}$. The conformal block on the other hand is usually defined [21] for the special choice $z_{i}=0, z, 1, \infty$. To define the correlator as the point $z_{4}$ moves to infinity we must rescale by a factor of $z_{4}^{2 h_{w}}$,

$\lim _{z_{4} \rightarrow \infty} z_{4}^{2 h_{w}} \bar{z}_{4}^{2 \overline{h_{v}}}\left\langle\prod_{i=1}^{4} \mathcal{O}_{i}\left(z_{i}\right)\right\rangle=\sum_{p} C_{12 p} C_{34 p} \mathcal{F}(p, z) \overline{\mathcal{F}}(p, \bar{z})$.
Comparing the two formulae yields

$$
\begin{aligned}
& \left.\lim _{z_{4} \rightarrow \infty} z_{4}^{2 h_{w}} \bar{z}_{4}^{2 \bar{h}_{v}}\left\langle\prod_{i=1}^{4} \mathcal{O}_{i}\left(z_{i}\right)\right\rangle\right|_{z_{1}=0, z_{3}=1, z_{2}=z} \\
& =f(z, \bar{z})(1-z)^{h / 3-h_{2}-h_{3}} z^{h / 3-h_{1}-h_{2}}
\end{aligned}
$$

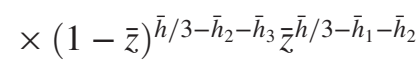

$$
\begin{aligned}
& =\sum_{p} C_{12 p} C_{34 p} \mathcal{F}(p, z) \overline{\mathcal{F}}(p, \bar{z}),
\end{aligned}
$$

and we see the canonical form of the 4-point function involves a nontrivial rescaling of the conformal block by a function of the cross-ratio.

Later when we study the commutator of two operators, $V$ and $W$ as a function of time, it will be convenient to factor out the norm of the operators. To accomplish this we compute

$$
\begin{aligned}
& \frac{\left\langle V\left(z_{1}\right) V\left(z_{2}\right) W\left(z_{3}\right) W\left(z_{4}\right)\right\rangle}{\left\langle V\left(z_{1}\right) V\left(z_{2}\right)\right\rangle\left\langle W\left(z_{3}\right) W\left(z_{4}\right)\right\rangle} \\
& \quad=z^{2 h_{v}} \bar{z}^{2 \bar{h}_{v}} \sum_{p} C_{12 p} C_{34 p} \mathcal{F}(p, z) \overline{\mathcal{F}}(p, \bar{z})
\end{aligned}
$$

using (A1) and (A3). Now the expression for general $z_{i}$ is a function only of the cross-ratios. Finally we note that in performing a coordinate transformation to a different coordinate system, each correlator of primaries transforms by

$$
\left\langle\prod_{i} \mathcal{O}\left(x_{i}\right)\right\rangle=\prod_{i}\left(\frac{\partial z}{\partial x}\right)_{z=z_{i}}^{h_{i}}\left(\frac{\partial \bar{z}}{\partial \bar{x}}\right)_{\bar{z}=\bar{z}_{i}}^{\bar{h}_{i}}\left\langle\prod_{i} \mathcal{O}\left(z_{i}\right)\right\rangle,
$$

and these factors cancel in the expression (A4).
[1] Y. Sekino and L. Susskind, Fast scramblers, J. High Energy Phys. 10 (2008) 065.

[2] D. A. Roberts and D. Stanford, Diagnosing Chaos Using Four-Point Functions in Two-Dimensional Conformal Field Theory, Phys. Rev. Lett. 115, 131603 (2015).

[3] A. L. Fitzpatrick, J. Kaplan, and M. T. Walters, Universality of long-distance AdS physics from the CFT bootstrap, J. High Energy Phys. 08 (2014) 145.

[4] S. H. Shenker and D. Stanford, Black holes and the butterfly effect, J. High Energy Phys. 03 (2014) 067.

[5] S. H. Shenker and D. Stanford, Stringy effects in scrambling, J. High Energy Phys. 05 (2015) 132.
[6] P. Caputa, J. Simón, A. Štikonas, T. Takayanagi, and K. Watanabe, Scrambling time from local perturbations of the eternal BTZ black hole, J. High Energy Phys. 08 (2015) 011.

[7] P. Hosur, X.-L. Qi, D. A. Roberts, and B. Yoshida, Chaos in quantum channels, J. High Energy Phys. 02 (2016) 004.

[8] C. T. Asplund, A. Bernamonti, F. Galli, and T. Hartman, Entanglement scrambling in $2 \mathrm{~d}$ conformal field theory, J. High Energy Phys. 09 (2015) 110.

[9] A. L. Fitzpatrick, J. Kaplan, and M. T. Walters, Virasoro conformal blocks and thermality from classical background fields, J. High Energy Phys. 11 (2015) 200. 
[10] Wolfram Research, Gauss hypergeometric function, series expansion, formula 07.23.06.0013.01, http://functions .wolfram.com/07.23.06.0013.01.

[11] A. L. Fitzpatrick, J. Kaplan, D. Li, and J. Wang, On information loss in $\mathrm{AdS}_{3} / \mathrm{CFT}_{2}$, J. High Energy Phys. 05 (2016) 109.

[12] A. L. Fitzpatrick and J. Kaplan, On the late-time behavior of Virasoro blocks and a classification of semiclassical saddles, J. High Energy Phys. 04 (2017) 072.

[13] T. Appelquist and J. Carazzone, Infrared singularities and massive fields, Phys. Rev. D 11, 2856 (1975).

[14] D. A. Lowe, The Planckian conspiracy: String theory and the black hole information paradox, Nucl. Phys. B456, 257 (1995).

[15] D. A. Lowe, J. Polchinski, L. Susskind, L. Thorlacius, and J. Uglum, Black hole complementarity versus locality, Phys. Rev. D 52, 6997 (1995).

[16] A. B. Zamolodchikov, Conformal symmetry in twodimensional space: Recursion representation of conformal block, Theor. Math. Phys. 73, 1088 (1987).
[17] We clarify that in most mathematical literature, the complete elliptic integral $K$ is defined with the modulus $k$ as the argument. Our $z$ is related to $k$ through $z=k^{2}$. It is also common for many mathematicians to use the symbol $m$ for our $z$.

[18] C. Bogner, A. Schweitzer, and S. Weinzierl, Analytic continuation and numerical evaluation of the kite integral and the equal mass sunrise integral, Nucl. Phys. B922, 528 (2017).

[19] R. Fan, Out-of-time-order correlation functions for unitary minimal models, arXiv:1809.07228.

[20] P. H. Ginsparg, Applied conformal field theory, in Les Houches Summer School in Theoretical Physics: Fields, Strings, Critical Phenomena Les Houches, France, 1988 (Amsterdam, North-Holland, 1988), pp. 1-168.

[21] A. A. Belavin, A. M. Polyakov, and A. B. Zamolodchikov, Infinite conformal symmetry in two-dimensional quantum field theory, Nucl. Phys. B241, 333 (1984). 